\title{
Caractérisation De La Sécheresse Climatique Du Bassin Versant D'oum Er Rbia (Maroc) Par Le Biais De L’indice De Précipitation Standardisé (SPI)
}

\author{
Yousra Daki \\ Ghalem Zahour \\ Laboratoire des Chaines Anciennes (LCA), \\ Département de Géologie,Faculté des Sciences Ben M'sik, \\ Université Hassan II de Casablanca - Maroc \\ Rahal Lachgar
}

Ingénieur météorologique, chef de service du contrôle des prévisions et du suivi des prestations, Direction de la météorologie national (DMN),

Casablanca - Maroc

Hassan El Hadi

Laboratoire des Chaines Anciennes (LCA),

Département de Géologie, Faculté des Sciences Ben M'sik,

Université Hassan II de Casablanca - Maroc

doi: 10.19044/esj.2016.v12n14p198 URL:http://dx.doi.org/10.19044/esj.2016.v12n14p198

\begin{abstract}
In recent decades, the drought becomes one of the important phenomena resulting from and climate change variability. Its indirect effects in the time can have an economic, agricultural, hydrological or social impact. The aim objective of this study is to characterize the climate drought at the catchment level of Oum Er Rbia (Morocco) for the period 1985/2013 through the calculation of the Standardized Precipitation Index (SPI) for the four stations studied: El Jadida, Safi, Beni Mellal and Khouribga. The Standardized Precipitation Index (SPI) originally proposed by Mckee et al. (1993) is used as an index of follow-up to the drought and recommended by the World Meteorological Organization. The results obtained after the calculation of this index have enabled us to locate the drought periods of more remarkable in their intensity, their duration and their frequency.
\end{abstract}

Keywords: Drought, climate change, standardized precipitation index (SPI), watershed Oum Er Rbia 


\section{Résumé}

La sécheresse est l'un des phénomènes importants résultant de la variabilité et du changement climatique des dernières décennies. Ses effets indirects dans le temps peuvent avoir des impacts économiques, agricoles, hydrologiques ou sociaux. L’objectif de cette étude est de caractériser la sécheresse climatique au niveau du bassin versant d’Oum Er Rbia (Maroc) pour la période 1985/2013 à travers le calcul de l'indice de précipitation normalisé (SPI) pour les quatre stations étudiées : El Jadida, Safi, Béni Mellal et Khouribga. L’indice de précipitation normalisé (SPI) proposé à l'origine par Mckee et al. (1993) est utilisé comme un indice de suivi de la sécheresse et recommandé par l’organisation météorologique mondiale. Les résultats obtenus après le calcul de cet indice nous ont permis de localiser des périodes de sécheresses plus remarquables de par leur intensité, leur durée et leur fréquence.

Mots clés : Sécheresse, changement climatique, indice de précipitation normalisé (SPI), bassin versant d’Oum Er Rbia

\section{Introduction}

Dans tous les pays de la Méditerranée, situés en zone subtropicale semi-aride, la pluviométrie est le paramètre climatique dominant, étant d'une part, généralement insuffisante, et, d’autre part, beaucoup plus variable que la température. Généralement, le changement climatique entraînera le déplacement des zones climatiques et la modification des régimes pluviométriques (GIEC, 2007). Par ailleurs, durant ces dernières décennies, le prolongement des épisodes secs est devenu une réalité dans cette partie du globe, notamment le Maroc (Sebbar et al., 2011).

Certains scénarios des changements planétaires indiquent que l'occurrence et l'impact des sécheresses risquent d'augmenter dans les années à venir (Watson et al., 1997). Dans un tel contexte climatique, il est indispensable de pouvoir analyser les séquences de sécheresse météorologique en vue de proposer aux populations des mesures d'atténuation ou d'adaptation dans le cas échéant. C’est dans ce cadre que la présente étude a été initiée dans le bassin versant d’Oum Er Rbia(Maroc).Cette région est une zone de forte production agricole et elle est sujette depuis plusieurs années à des déficits pluviométriques.

Selon une échelle plus large, la variabilité du climat est influencée par les changements de la circulation atmosphérique générale, qui comprend des situations météorologiques à diverses échelles temporelles (Khomssi, 2014): jours (système frontaux) ; semaines (périodes sèches) ; mois (hivers froids); années (succession des étés chauds et sécheresse); siècles (fluctuations et changements climatiques). 
Plusieurs régions du Maroc ont été confrontées à des séries de périodes sèches depuis le début des années 1980 et qui ont eu des conséquences non négligeables sur l'économie du pays (Driouech, 2010).

Différentes études de projections climatiques futures, faisant appel à des modèles climatiques, préconisent que le Maroc est l'un des pays les plus susceptibles à être menacé par le changement climatique et enregistrera une réduction des cumuls pluviométriques d'ici la fin du siècle courant (IPCC, 2007a).

En effet, le climat du Maroc s’est réchauffé de façon significative au cours de la période 1961-2008. Une tendance générale vers l'assèchement est constatée. La plupart des tendances annuelles sont comprises entre $0.2^{\circ} \mathrm{C}$ et $0.4{ }^{\circ} \mathrm{C}$ par décennie (Driouech, 2010) et la diminution des précipitations est quasi-générale sur l'ensemble du territoire marocain. D’autres études signalent que durant la phase positive de l'ONA (Oscillation Nord Atlantique) caractérisée par le creusement de la dépression d'Islande et gonflement/intensification de l'anticyclone des Açores (Fig.2), les précipitations se trouveront réduites sur le Maroc comme sur le sud de l'Europe. A l'inverse, la phase négative engendrera des précipitations sur celui-ci et au Nord-Ouest de l'Afrique (Driouech, 2010).

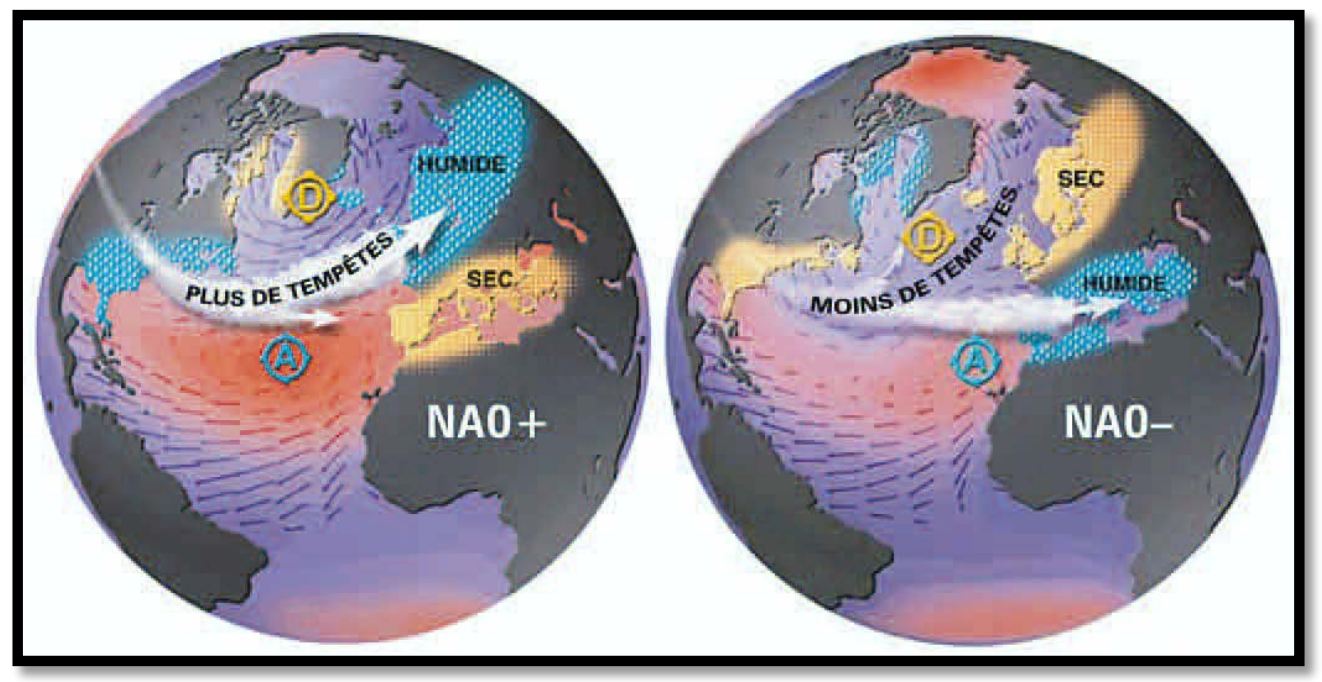

Fig.2 : les deux phases de la NAO (Christophe, 2004).

Selon (Sebbar, 2013), la relation entre l'ONA et les valeurs de l'indice de précipitation standardisé, a permis d'émettre des hypothèses qui expliquent l'extension spatio-temporelle de la sécheresse climatique au Maroc. En fait, l'excès d'énergie au niveau de la surface du pacifique (ENSO), renforce l'extension de l'anticyclone des Açores à travers la cellule de Hadley et par conséquent, rejette vers le Nord des perturbations liées au 
front polaire et apparition des phases positives de l'ONA, ce qui explique l'installation de la sécheresse au Maroc.

\section{Présentation de la zone d'étude}

\section{Situation géographique}

Le bassin de l'Oum-Er-Rbia(Fig.3) s'étend sur une superficie de $33.520 \mathrm{~km}^{2}$ (Ciobotaru, 2014). L’Oued Oum Er Rbia, d’une longueur de 550 km, prend son origine au Moyen Atlas à 1800 m d'altitude, traverse la chaîne du Moyen Atlas, la plaine du Tadla et la Meseta côtière et se jette dans l'Océan Atlantique à environ $16 \mathrm{~km}$ au Nord de la ville d'El Jadida.

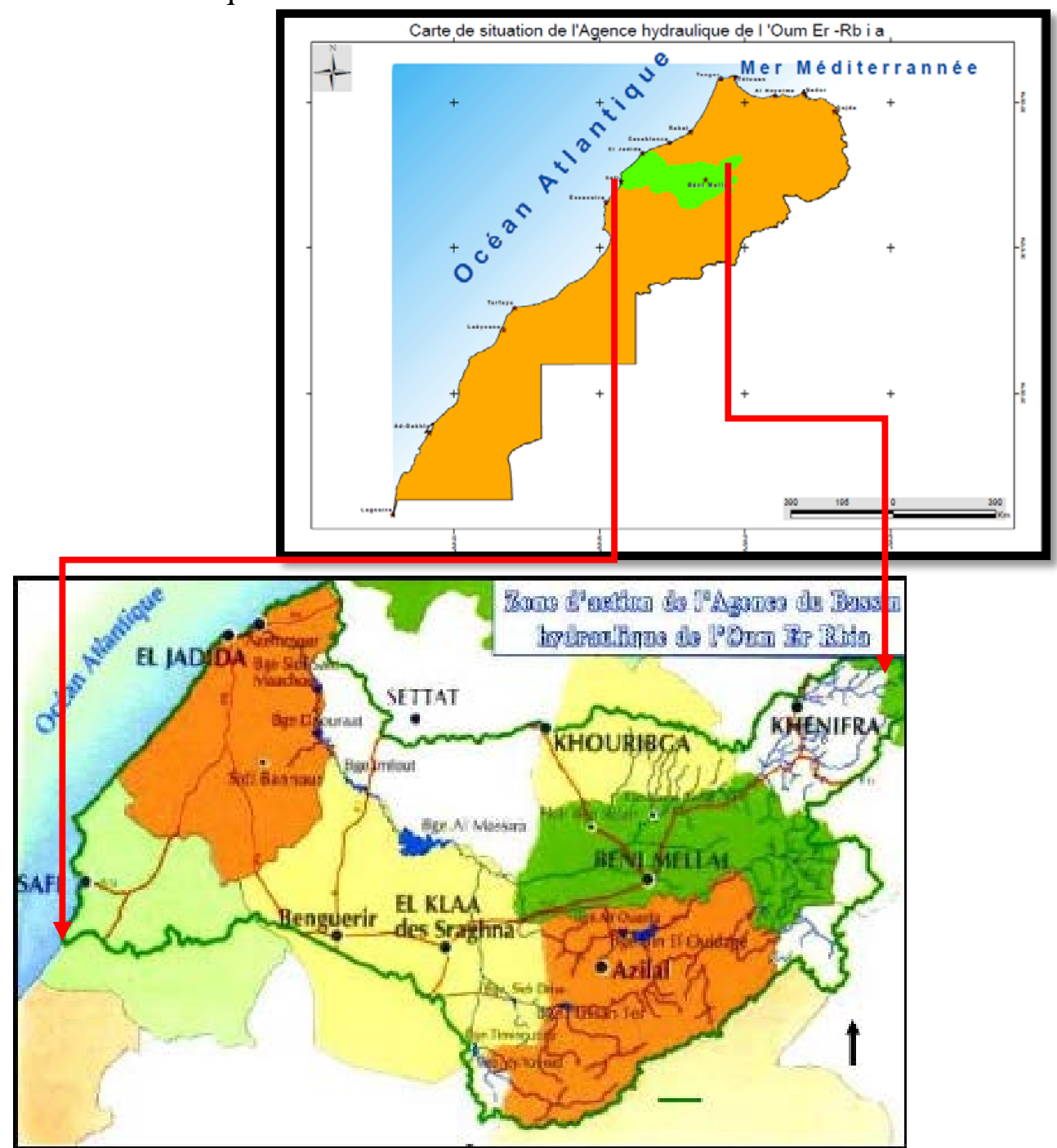

Fig.3 : Situation géographique du bassin versant d’Oum Er Rbia (ABHOER, 2010) 
Le bassin, comme la montre, la figure ci-dessus, est délimité par (ABHOER, 2010) :

- $\quad$ Le bassin de Bouregreg au Nord ;

- $\quad$ L'océan atlantique à l'Ouest ;

- $\quad$ Les bassins de Tensift et de Draa au Sud ;

- $\quad$ Les bassins du Ghris et Moulouya à l’Est ;

- $\quad$ Le bassin du Sebou au Nord Est.

\section{Contexte géologique}

La zone d'étude fait partie du domaine Atlantique. Elle s’étend sur six unités géographiques (ABHOER, 2010):

- Le massif des Rhamna constitué par des formations sédimentaires paléozoïques fortement plissées avec une couverture d’âge secondaire sur les bordures ;

- Le plateau des phosphates formé d'un ensemble de plates-formes emboîtées correspondant chacune aux niveaux calcaires les plus résistants de la série sédimentaire. Il est caractérisé par l'existence du gisement de phosphates d'Ouled Abdoun ;

- La zone des Abda-Doukkala qui fait partie de la Méséta marocaine. Cette dernière est caractérisée par un régime tabulaire de dépôts secondaires et tertiaires reposant sur des terrains primaires plissés ;

- La zone côtière d'Azemmour à Safi qui présente une morphologie dunaire avec une succession de crêtes séparées de dépressions remplies de limons très sableux ;

- La plaine de Tadla constituée d'une vaste dépression asymétrique recouverte de dépôts hétérogènes. Cette plaine est caractérisée par l'existence de deux importants périmètres agricoles, situés de part et d'autre de l’Oued Oum Rbia (Béni Amir et Béni Moussa);

- Le plateau des Gantour, et les plaines de Bhira et Tassaout aval le socle primaire est recouvert par des formations de couverture triasiques, crétacées et éocènes, antérieurs aux premiers mouvements atlasiques. Ces formations affleurent au Nord et s'enfoncent sous la plaine vers le Sud. Elles sont recouvertes par des formations continentales de remplissage récent et par des dépôts quaternaires.

\section{Cadre climatique}

La zone d'étude peut être divisée en zones homogènes à savoir (ABHOER, 2010):

\footnotetext{
$>\quad$ TS (Oued Tessaout). AB (Oued El Abid);

$>\quad$ HO (Haut Oum Er Rbia), CO (Oum Er Rbia central);

$>\quad$ MO (Moyen Oum Er Rbia), BO (Bas Oum Er Rbia);
} 
$>\quad$ LT (Limitrophe Oum Er Rbia-Tensift).

Le bassin d'Oum Er Rbia(ABHOER, 2010) présente des zones à climats différents allant du climat littoral modéré qui caractérise la zone côtière au climat aride caractérisant la plaine de Rhamna en passant par le climat semi-aride dans la plaine de Tadla et des zones plus arrosées en montagne. Le bassin a connu une diminution de la pluviométrie. La pluviométrie moyenne du bassin est passée d'environ $400 \mathrm{~mm} / \mathrm{an}$ au cours de la période 1935 - 1980, à 340 mm/an au cours de la période 1980 -2007.

L’objectif de cette étude est de caractériser la sécheresse climatique au niveau du bassin versant d'Oum Er Rbia, pour la période 1985/2013, à travers le calcul de l'indice de la précipitation normalisé (spi), calculé à partir d'une série pluviométrique de 30 ans. On a choisi pour cette étude quatre stations(Fig.4): El Jadida, Safi, Khouribga et Béni Mellal.

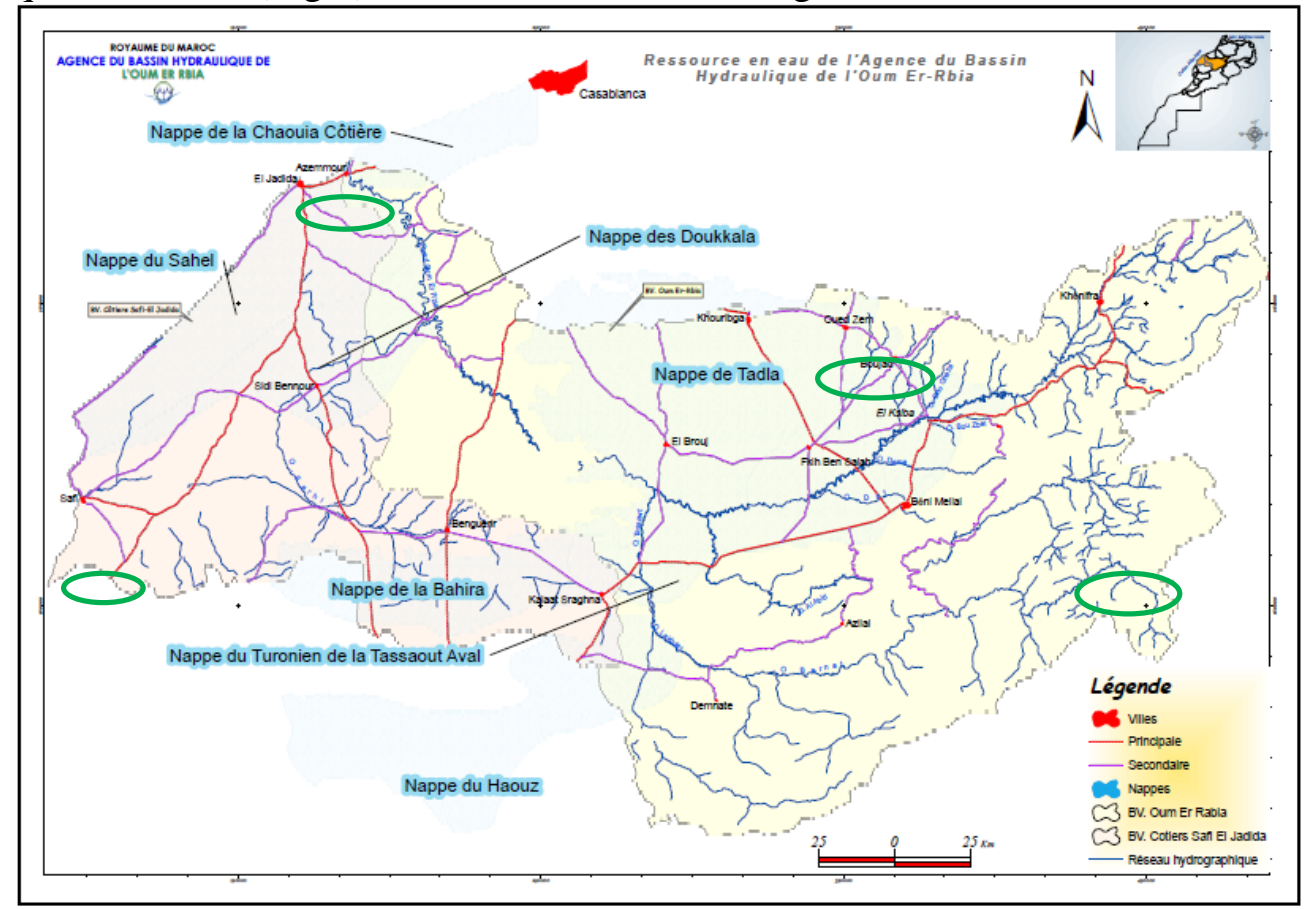

Fig.4 : Carte de situation des stations pluviométriques (ABHOER, 2010)

Les données pluviométriques qui ont été collectées et exploitées dans cette étude, proviennent de la Direction de la Météorologie Nationale(DMN). Les caractéristiques des différentes stations utilisées dans cette étude sont données dans le tableau 1: 
Tableau 1 : Caractéristiques des différentes stations pluviométriques

\begin{tabular}{|cccccccc|} 
Nom de station & Latitude & Longitude & Altitude & $\begin{array}{c}\text { Taille } \\
\text { des } \\
\text { séries } \\
\text { (ans) }\end{array}$ & $\begin{array}{c}\text { PMA } \\
(\mathrm{mm})\end{array}$ & $\begin{array}{c}\text { Ecart } \\
\text { type }\end{array}$ & $\begin{array}{c}\mathrm{CV} \\
(\%)\end{array}$ \\
\hline SAFI & $32^{\circ} 17^{\prime} \mathrm{N}$ & $9^{\circ} 14^{\prime} \mathrm{O}$ & $43,0 \mathrm{~m}$ & 30 & 333,4 & 116,3 & 35 \\
\hline EL JADIDA & $33^{\circ} 14^{\prime} \mathrm{N}$ & $8^{\circ} 31^{\prime} \mathrm{O}$ & $27,0 \mathrm{~m}$ & 30 & 369,0 & 155,6 & 42 \\
\hline KHOURIBGA & $32^{\circ} 52^{\prime} \mathrm{N}$ & $6^{\circ} 58^{\prime} \mathrm{O}$ & $770,9 \mathrm{~m}$ & 30 & 318,7 & 123,4 & 39 \\
\hline $\begin{array}{c}\text { BENI } \\
\text { MELLAL }\end{array}$ & $32^{\circ} 22^{\prime} \mathrm{N}$ & $6^{\circ} 24^{\prime} \mathrm{O}$ & $468,0 \mathrm{~m}$ & 30 & 324,7 & 110,8 & 34 \\
\hline
\end{tabular}

\section{Matériel et méthodes}

\section{Indice normalisé(Standardisé) des précipitations(SPI)}

$>\quad$ Traitement des données pluviométriques

Au fil des ans, les météorologues et les climatologues ont mis au point partout dans le monde de nombreux indices de sécheresse, allant des plus simples, notamment le pourcentage de la normale des précipitations ou les centiles de précipitations, aux plus complexes, tel que l'indice de sécheresse de Palmer. Aux États-Unis d'Amérique, des spécialistes de la question ont pris conscience du fait qu'un indice devait être simple, facile à calculer et statistiquement adapté et significatif. De plus, la compréhension des différents effets que peuvent avoir des déficits de précipitations sur les eaux souterraines, sur le volume stocké dans les réservoirs, sur l'humidité du sol, sur le manteau neigeux et sur l'écoulement des cours d'eau, a conduit les scientifiques américains McKee, Doesken et Kleist à mettre au point, en 1993, l’indice de précipitations normalisé (SPI).

L’indice SPI (Mckee et al., 1993) est un indice à la fois puissant, souple d'utilisation et simple à calculer. Les données sur les précipitations constituent en fait le seul paramètre requis. En outre, l'indice SPI se révèle tout aussi efficace pour analyser les périodes ou cycles humides que les périodes ou cycles secs (tableau2). Le programme de calcul de l'indice s'exécute aussi bien dans un environnement Windows que sous UNIX.

Pour calculer l'indice SPI, il faut disposer idéalement de relevés mensuels s'étalant sur au moins 20 à 30 ans, mais de préférence sur 50 à 60 ans, voire plus, ce qui constitue la période optimale (Guttman, 1994).

\section{Description de l’indice normalisé de précipitations}

L'indice SPI (MCKEE et al., 1993) est fondé sur la probabilité de précipitations sur un laps de temps donné. La probabilité des précipitations observées est transformée en un indice qui sert aussi bien à l'expérimentation qu'à l'exploitation dans plus de 70 pays (tableau3).

Les responsables de la planification en matière de sécheresse sont nombreux à apprécier la souplesse d'utilisation de l'indice SPI. Ce dernier 
est utilisé aussi dans divers instituts de recherche, des universités et des services météorologiques et hydrologiques nationaux du monde entier, dans le cadre d’activités de suivi de la sécheresse et d’alerte précoce dans le domaine.

Avantages: Les précipitations représentent le seul paramètre dont il faut disposer. Il est possible de calculer l'indice pour diverses échelles de temps, celui-ci permettant de détecter rapidement les situations de sécheresse et d'en évaluer la gravité. Il est moins complexe que bien d'autres indices et notamment l'indice de sécheresse de Palmer.

Inconvénients: Il ne permet de quantifier que le déficit de précipitations; les valeurs basées sur des données préliminaires peuvent changer et les valeurs varient si la durée de la période des relevés s’allonge.

Tableau2 : Classe de sévérité de sécheresse selon (Mckee et al., 1993)

\begin{tabular}{|cc|}
\hline Valeurs de l'indice SPI & Type de sècheresse \\
\hline 2,0 et plus & Extrêmement humide \\
\hline de 1,5 à 1,99 & Très humide \\
\hline de 1,0 à 1,49 & Modérément humide \\
\hline de $-\mathbf{0 , 9 9}$ à 0,99 & Proche de la normale \\
\hline de $-\mathbf{1 , 0}$ à $-\mathbf{1 , 4 9}$ & Modérément sec \\
\hline de $-\mathbf{1 , 5}$ à $\mathbf{- 1 , 9 9}$ & Très sec \\
\hline-2 et moins & Extrêmement sec \\
\hline
\end{tabular}

Tableau3 : Probabilité d’occurrence (Mckee et al., 1993)

\begin{tabular}{|c|c|c|c|}
\hline Indice SPI & Catégorie & $\begin{array}{l}\text { Nombre de fois } \\
\text { sur } 100 \text { ans }\end{array}$ & Fréquence \\
\hline de 0 à $-0,99$ & Légèresécheresse & 33 & 1 fois tousles 3 ans \\
\hline de $-1,00$ à $-1,49$ & Sécheressemodérée & 10 & 1 fois tousles 10 ans \\
\hline de $-1,5$ à $-1,99$ & Grandesécheresse & 5 & 1 fois tousles 20 ans \\
\hline$<-2,0$ & Sécheresseextrême & 2 & 1 fois tousles 50 ans \\
\hline
\end{tabular}

\section{Résultats et discussions}

Au niveau du bassin versant d’Oum Er Rbia, l’évolution des degrés de sévérité de la sécheresse a été analysée en utilisant l'indice de précipitation standardisé calculé à partir d'une série pluviométrique de 30 années, au cours de la période 1985-2013.

L'analyse des résultats de l'indice pluviométrique standardisé à la station de Béni Mellal (Fig.5) montre qu’on a une dominance des années sèches bien remarquable entre la période (1985-1995), on note aussi que l'indice spi entre cette période peut atteindre (-3), ce qui explique l'existence des années extrêmement sèches.

Autour des années 1996, 2002 et 2008, on note des années extrêmement humides, puis après 2010, on remarque un assèchement des conditions climatiques. 


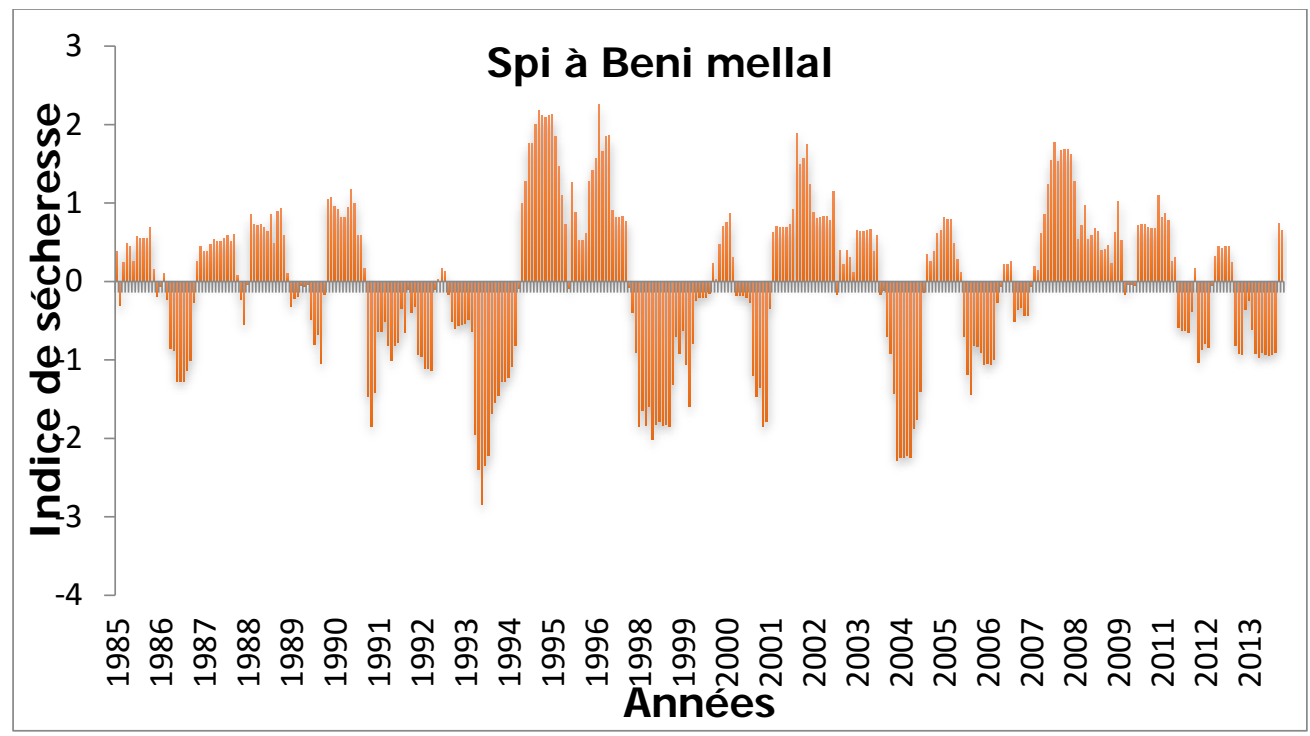

Fig.5 : Indice pluviométrique standardisé à la station de Béni Mellal au cours de la période1985/2013

L'analyse des résultats de calcul de l'indice spi à la station de Safi (Fig.6), nous a permis de confirmer que la période entre 1985-1997 est caractérisée par une alternance des années sèches et des années humides, pour la période de 1997 à 2001, les résultats montre des années très sèches, puis après cette date, on a une alternance entre des années modérément sèches et des années humides à extrêmement humides.

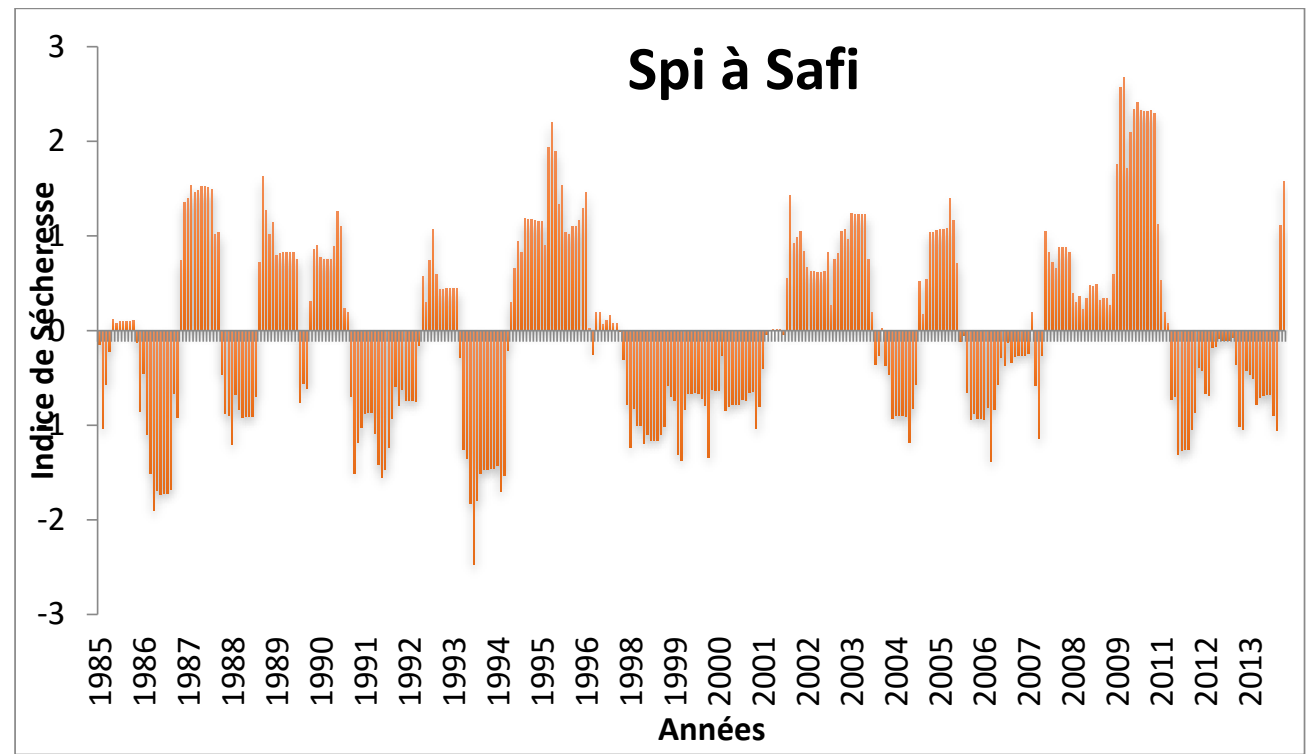

Fig.6 : Indice pluviométrique standardisé à la station de Safi au cours de la période 1985/2013 
A partir du 1985 jusqu'à 1995, les valeurs de l'indice pluviométrique standardisé(Fig.7) au niveau de la station d'El Jadida, sont négatives et montrent des années extrêmement sèches, après cette date, on a remarqué la dominance des années normales.

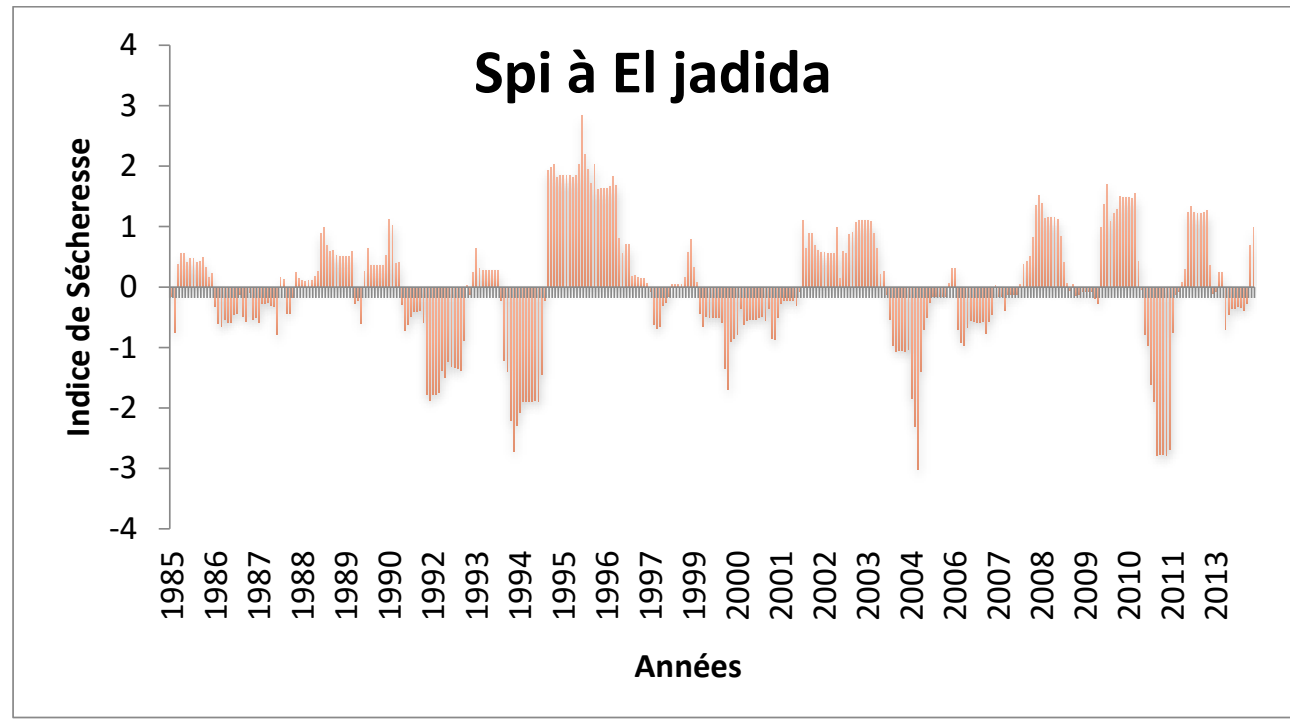

Fig.7 : Indice pluviométrique standardisé à la station d’El Jadida au cours de la période1985/2013

Les résultats de l'indice de précipitation standardisé à la station de Khouribga(Fig.8) indiquent globalement une sécheresse extrême pendant toute la période d'étude, sauf quelques exceptions comme les années 1996, 2002, 2003,2008, 2009, et 2010.

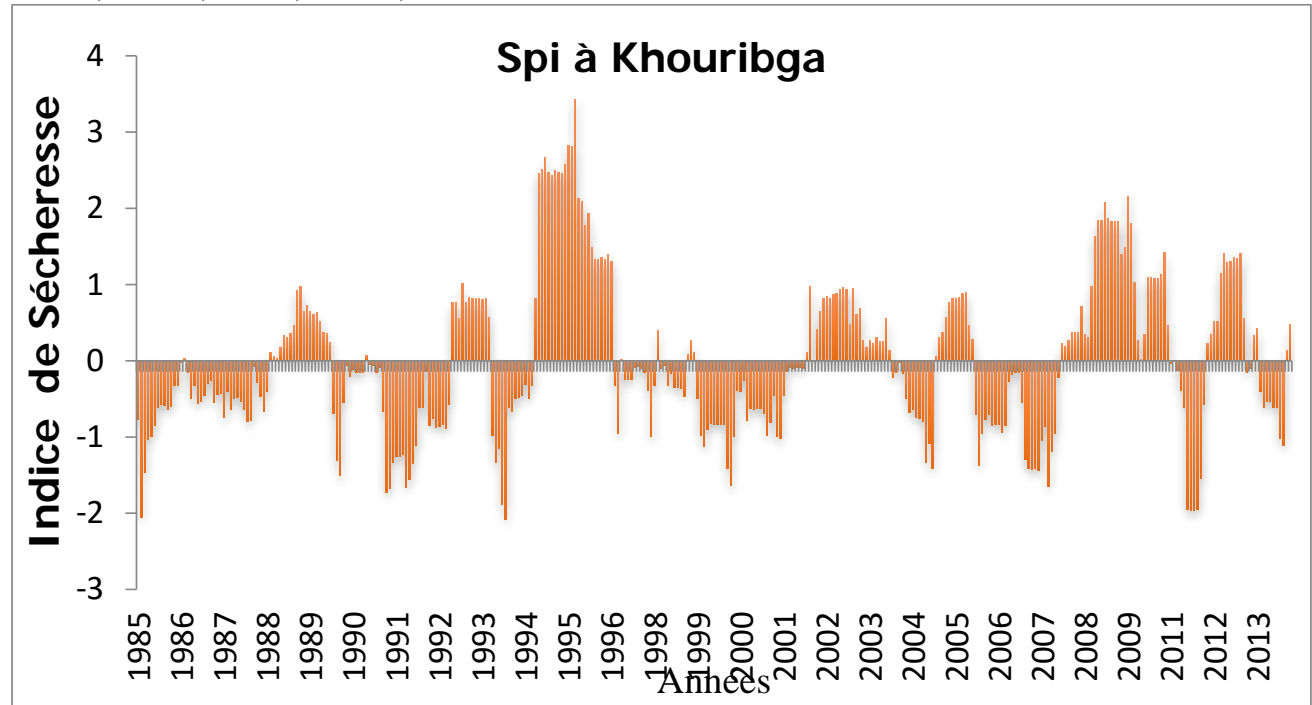

Fig.8 : Indice pluviométrique standardisé à la station de Khouribga au cours de la période1985/2013 
A l'échelle mensuelle, les résultats montrent l'existence de nombreuses séquences sèches tout au long de la période analysée, l'indice SPI a permis de caractériser leur sévérité, mais il est important de souligner l'existence de deux séquences (tableau4) dont la durée égale ou dépasse 24 mois, la plus longue se situe entre les années 1998-2001.

Tableau4 : Séquences sèches dépassant l'année dans le Bassin versant d’Oum Er Rbia

\begin{tabular}{|cccc|}
$\begin{array}{c}\text { Séquences } \\
\text { Sèches }\end{array}$ & $\begin{array}{c}\text { Durée } \\
\text { (mois) }\end{array}$ & $\begin{array}{c}\text { Valeurs de l'indice Spi Intensité } \\
\text { maximale }\end{array}$ & $\begin{array}{c}\text { Type de } \\
\text { sécheresse }\end{array}$ \\
\hline $\mathbf{1 9 9 8 - 2 0 0 1}$ & 30 & $-1,19$ & Modérément sec \\
\hline $\mathbf{2 0 0 7 - 2 0 0 8}$ & 24 & $-1,00$ & Modérément sec \\
\hline
\end{tabular}

\section{Conclusion}

Au terme de ce travail, nous retenons que le bassin versant d'Oum Er Rbia a été affecté par plusieurs séquences sèches sur la période 1985 à 2013.

Les résultats obtenus après le calcul de l'indice de précipitation standardisé, nous ont permis de localiser des séquences de sécheresses dans les quatre stations étudiées dans lebassin :

- $\quad$ Lastation d'El Jadida : à partir du 1985 jusqu'à 1995, on remarque des années modérément sèches ;

- $\quad$ Lastation de Safi : pour la période de 1997 à 2001, les résultats montrent des années très sèches ;

- $\quad$ La station de Béni mellal : pour la période de 1991à 1995, l’indice spi montre des années extrêmement sèches ;

- $\quad$ La station de Khouribga : la plupart des années de la période d'étude sont très sèches.

\section{References:}

ABHOER, Ministère de l'aménagement du territoire, de l'eau et de l'environnement, (2010). Étude d'actualisation de la zone d'action de l'agence du bassin hydraulique de l'Oum Er Rbia, Rapport d'étude définitif sur l'état des lieux et perspective d'évolution, $43 \mathrm{p}$.

CIOBOTARU A., (2014). Les changements climatiques et environnementaux du bassin de l'Oum Er Rbia(Maroc). Conference paper, May 2014. ResearchGate

CRISTOPHE C., (2004). Du changement climatique aux régions de temps : l’Oscillation Nord Atlantique. La Météorologie - n45.21-32p.

DRIOUECH F., (2010). Distribution des précipitations hivernales sur le Maroc dans le cadre d'un changement climatique. Thèse de Doctorat de l'Institut national polytechnique de Toulouse, $163 \mathrm{p}$.

GIEC, 2007 : Bilan 2007 des changements climatiques. Contribution des Groupes de travail I, II et III au quatrième Rapport d'évaluation du Groupe d'experts intergouvernemental sur l'évolution du climat [Équipe de rédaction 
principale, Pachauri, R.K. et Reisinger, A. (publié sous la direction de )]. GIEC, Genève, Suisse... 103 pages.)

Guttman N.B., 1994. On the sensitivity of sample L moments to sample size. Journal of Climate, 7(6):1026-1029.

KHOMSI K., 2014.Variabilité hydroclimatique dans les bassins versants du Bouregreg et du Tensift au Maroc: moyennes, extrêmes et projections climatiques. Thèse de doctorat en climatologie, Univ. Mohammed V, Fac. Sci.Rabat, 218p.

McKee, T.B., N.J. Doeskenet J. Kleist, (1993): The relationship of drought frequency and duration to time scale. In: Proceedings of the Eighth Conference on Applied Climatology, Anaheim, California, du 17 au 22 janvier 1993. Boston, American Meteorological Society, 179-184.

IPCC, (2007a): The Physical Science Basis. Contribution of Working Group I to the Fourth Assessment Report of the Intergovernmental Panel on Climate Change [Solomon, S., D. Qin, M. Manning, Z. Chen, M. Marquis, K.B. Averyt, M.Tignor and H.L. Miller (eds.)]. Cambridge University Press, Cambridge, United Kingdom and New York, NY, USA, 996 p.

SEBBAR A., (2013). Etude de la variabilité et de l'évolution de la pluviométrie au Maroc (1935-2005): Réactualisation de la carte des précipitations. Thèse de Doctorat en Bioclimatologie, Univ. Hassan II, Fac. Sci. Ben M’sik, Casablanca, 186 p.

SEBBAR A, BADRI W, FOUGRACH H, HSAINE $M$ et SLAOUI A, (2011). Etude de la variabilité du régime pluviométrique au Maroc septentrional (1935-2004), Sécheresse vol. 22, n³ 3, pp48-139.

Watson,R.T.,M.C.ZinyoweraandR.H.Moss,Eds., (1997):The Regional Impacts of Climate Change: An Assessment of Vulnerability. Cambridge University Press, Cambridge, 517 pp. 